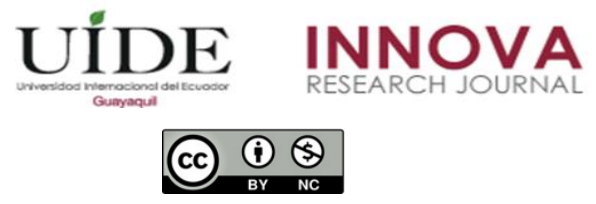

INNOVA Research Journal, ISSN 2477-9024

(Septiembre- Diciembre 2020). Vol. 5, No.3.1 pp. 115-184

DOI: https://doi.org/10.33890/innova.v5.n3.1.2020.1562

URL: http://revistas.uide.edu.ec/index.php/innova/index

Correo: innova@uide.edu.ec

\title{
Evaluación de la gestión financiera y cualidades gerenciales en empresas ecuatorianas
}

\section{Evaluation of financial management and managerial qualities in Ecuadorian companies}

Gabriela Duque Espinoza

(O) https://orcid.org/0000-0003-3783-1791

Fernando Córdova León

(D)https://orcid.org/0000-0002-7511-688X

Karla González Soto

(D) https://orcid.org/0000-0001-9725-880

Juan Carlos Aguirre Quezada

(D)https://orcid.org/0000-0002-8953-8151

Universidad del Azuay, Ecuador

Autor para correspondencia: gduque@uazuay.edu.ec; jfcordova@uazuay.edu.ec;

kngonzalez@uazuay.edu.ec; jcaguirre@uazuay.edu.ec

Fecha de recepción: 21 de septiembre de 2020 - Fecha de aceptación: 06 de noviembre de 2020

\section{Resumen}

Los recursos que disponen las empresas y los resultados que obtienen, determinan su situación financiera y desempeño económico, reflejados en sus estados financieros. La función gerencial ejerce un rol fundamental para la maximización del valor empresarial, mediante el liderazgo, dirección y la acertada toma de decisiones; de forma que, las cualidades de la persona que administra una empresa repercuten en sus resultados. Esta investigación tiene como objetivo evaluar la gestión financiera de los cuatro sectores económicos más importantes de la industria manufacturera del Ecuador: Cárnico, Textil, Bebidas y Muebles, durante el período 2013 - 2018, y estudiar su relación con la gerencia, a través de la cuantificación y análisis de sus cualidades, tales como género, permanencia en el cargo, formación académica y afinidad. Se utilizaron los estados financieros reportados por las empresas ante la Superintendencia de Compañías, Valores y Seguros del Ecuador. La gestión financiera se evaluó mediante la aplicación y comparación de razones financieras, con la finalidad de estudiar su comportamiento y evolución, al identificar las principales similitudes y diferencias. Los resultados muestran una participación mayoritaria del género masculino en la gerencia de los cuatro sectores estudiados. El índice de endeudamiento es superior cuando la gerencia posee formación universitaria, lo que evidencia que la preparación académica incide en la capacidad de asumir mayores riesgos. Las empresas cuyos gerentes poseen formación afín, presentan mayores niveles de liquidez, lo que muestra mejores estrategias financieras de corto plazo.

Palabras claves: gestión financiera; cualidades gerenciales; desempeño económico; razones financieras; género. 


\begin{abstract}
The resources available to companies and the results they obtain determine their financial situation and economic performance, reflected in their financial statements. The managerial function plays a fundamental role for the maximization of business value, through leadership, direction, and correct decision-making; so that, the qualities of the person who manages a company have an impact on its results. This research aims to evaluate the financial management of the four most important economic sectors of the Ecuadorian manufacturing industry: meat, textile, beverage and furniture, during the period 2013 - 2018, and to study their relationship with management, through the quantification and analysis of their qualities, such as gender, permanence in office, academic training and affinity. The financial statements reported by the companies to the Superintendency of Companies, Securities and Insurance of Ecuador were used. Financial management was evaluated by applying and comparing financial ratios, to study its behavior and evolution, by identifying the main similarities and differences. The results show a majority participation of the male gender in the management of the four sectors studied. The indebtedness index is higher when management has a university education, which shows that academic preparation affects the ability to take greater risks. Companies whose managers have related training have higher levels of liquidity, which shows better short-term financial strategies.
\end{abstract}

Keywords: financial management; managerial qualities; economic performance; financial ratios; gender.

\title{
Introducción
}

El aprovechamiento de los factores productivos mediante la interacción con el entorno determina los resultados y desempeño de las empresas; por tanto, su actividad eficiente y eficaz beneficia de forma directa a la sociedad en su conjunto, al ser las empresas generadoras de fuentes de empleo y valor económico agregado (Terrazas, 2016). La importancia del tejido empresarial en la economía ecuatoriana se refleja, entre otras variables, en el empleo formal; es así, que para el año 2019, de cada 100 plazas de empleo formal, 92 corresponden al sector privado (Instituto Nacional de Estadísticas y Censos, 2020).

Ecuador ha mantenido durante la última década niveles significativos de iniciativa emprendedora reflejada en la Tasa de Actividad Emprendedora Temprana más alta de la región de América Latina y el Caribe, desde el año 2012 hasta el 2019; sin embargo, esta situación contrasta con insuficientes tasas de perdurabilidad empresarial respecto a los numerosos emprendimientos nacientes (Global Entrepreneurship Monitor, 2020). La reducida supervivencia empresarial se relaciona con la inexistencia de planificación y gestión estratégica, baja rentabilidad y liquidez (Zambrano y Lasio, 2019); a su vez, el desempeño empresarial depende del éxito en la toma de decisiones gerenciales, las cuales son influenciadas positivamente por el acceso equitativo a información relevante, oportuna, confiable y de calidad (Zabukovec y Jaklič, 2015).

En un contexto de incertidumbre y constante cambio, es necesario proveer a los gerentes, inversionistas, el estado y sociedad en general, de información objetiva, oportuna y confiable que evalúe la gestión financiera y el desempeño económico de las empresas e industrias, con la finalidad de sustentar efectivamente el proceso de toma de decisiones, con la finalidad de elevar 
la competitividad del sector empresarial ecuatoriano. Por ello, el objetivo de la investigación es evaluar la gestión financiera de los cuatro sectores económicos más importantes de la industria manufacturera del Ecuador: Cárnico, Textil, Bebidas y Muebles, durante el período 2013 - 2018, y estudiar su relación con la gerencia, a través de la cuantificación y análisis de sus cualidades, tales como género, permanencia en el cargo, formación académica y afinidad.

El estudio, en primera instancia, presenta el marco teórico y las investigaciones más relevantes respecto al desempeño empresarial, la gestión financiera y su evaluación; así como, la importancia de las cualidades gerenciales sobre el desempeño económico empresarial. Posteriormente, se describe la información analizada y las técnicas y métodos empleados durante la investigación; para presentar los hallazgos más preponderantes y la discusión de los resultados obtenidos respecto a la literatura existente, lo cual permite plantear las conclusiones de la investigación.

\section{Estado del arte y Marco Teórico}

El análisis financiero es fundamental al momento de evaluar el desempeño económico de las empresas; de esta forma, es posible detectar problemas o irregularidades y actuar sobre ellos; además, de ser una fuente de comparación con otras empresas y sectores económicos. El análisis financiero debe ser aplicado por cualquier organización sin distinción de su tamaño o sector industrial; a través de este, se obtiene información valiosa que permite no solo un análisis que sustente la toma de decisiones, sino también examinar la situación real de la empresa desde una óptica distinta (Nava, 2009).

Al momento de analizar el desempeño empresarial en cualquier sector industrial es necesario estudiar la administración financiera, esta tiene el objetivo de guiar a una empresa hacia su óptimo de productividad y generación de valor; a través, de cuatro etapas: planificación, control, soporte de decisiones y evaluación del riesgo (Danvers y Oliver, 2013). La optimización no está relacionada con la actividad económica de la empresa, pues es una búsqueda de cualquier compañía en distintos ámbitos de comercio y producción; por lo tanto, al definir la gestión económica y financiera como un conjunto de procesos relacionados con la planificación, obtención y control de recursos, se consideran de forma implícita las decisiones necesarias para obtener rentabilidad y liquidez dentro de una operación empresarial saludable (Ramírez-Urquidy et al., 2018).

Según un enfoque tradicional, una organización tiene como objetivo la obtención y maximización de beneficios; sin embargo, este postulado puede llevar a generar desigualdades económicas entre varias de las partes involucradas. El enfoque moderno no busca la maximización de riqueza, sino la maximización de valor, considerando al valor como trascendental para todos los actores que se relacionan con la empresa (Paramasivan y Subramian, 2012).

La administración financiera se ocupa de la obtención y uso eficaz del dinero, para esto realiza actividades referentes a adquisición, inversión y administración de recursos; por ello, se afirma que las finanzas establecen actividades, procesos, técnicas y criterios que permiten optimizar la forma de obtener y usar estos recursos financieros (Besley y Brigham, 2001; Córdoba, 2012). Dentro de las prácticas de la gestión financiera se encuentran la reducción del 
costo del capital por medio de distintas fuentes de apalancamiento externas, el aumento de rentabilidad y la gestión del riesgo como vías para el incremento del valor presente de los activos (Pardo y Peña, 2018).

La evolución histórica que ha atravesado la administración financiera, hasta llegar a su estado actual, puede resumirse en tres periodos, enfatizando que cada uno de ellos llevó a que las técnicas utilizadas sean cada vez más efectivas y permitan a las empresas lograr su máximo potencial. En el primer periodo, se consideraban únicamente la solvencia y liquidez sin importar las decisiones empresariales como uno de sus objetivos. Para el segundo periodo, generado al finalizar la Segunda Guerra Mundial, los objetivos esenciales incluyeron el crecimiento y diversificación internacional, esto conllevó analizar las imperfecciones del mercado y prestar especial atención al momento de seleccionar activos, obtener financiamiento y repartir dividendos. En el último periodo, al abrirse nuevas líneas investigativas, se presentan avances en materia de valoración de empresas y el efecto que causa las imperfecciones e incertidumbre del mercado en el valor de estas (Córdoba, 2012).

Dentro de la administración financiera, Van Horne y Wachowicz (2010), indican que se deben utilizar razones o índices que evalúen la condición y desempeño de la empresa basándose en la información financiera que se obtiene de los estados financieros de cada compañía. De igual manera, Pacheco et al.,(2002) señalaron que los indicadores financieros constituyen el resultado de las prioridades financieras; es así, como permiten conocer la situación de la empresa en aspectos como liquidez, solvencia, eficiencia operativa, endeudamiento y rentabilidad.

Bernal y Amat (2012) proponen una publicación anual de ratios financieros por empresa y promedio sectorial en México, debido a la limitación de información financiera existente, y la relevancia de esta. Describen los índices financieros ideales con mayor potencial predictivo y sus fórmulas, elaboradas por un número importante de investigadores a nivel internacional. Se determinaron 43 indicadores que evalúan la liquidez, endeudamiento, gestión de activos, ciclo económico, rendimiento y capacidad de autofinanciación, con el fin de que los empresarios monitoreen sus indicadores con el promedio de la industria, lo cual será de gran utilidad para su planificación y toma de decisiones. La toma de decisiones ineficientes, pérdida de valor de la empresa o incumplimiento de objetivos pueden ser consecuencia de no realizar planificación financiera (Masilo y Gómez, 2016).

El desempeño de las empresas, considerando el sector económico al que pertenecen, ha sido evaluado por medio de la gestión financiera. Portal et al., (2016) evalúan el nivel de gestión económico-financiera en microempresas mexicanas y su impacto sobre los resultados, mediante un índice construido a través de la metodología de indicadores sintéticos, aplicando análisis factorial por componentes principales y ejercicios de regresión lineal. Como resultado se concluyó que, a medida que se incorporen elementos que permitan evaluar la gestión económica y financiera en las organizaciones, el impacto en los resultados será positivo por su pronta gestión.

Morelos et al., (2012) evaluaron el comportamiento de los indicadores financieros en las empresas del sector de alimentos de Barranquilla, Colombia. La descripción detallada de la evaluación de los sistemas organizacionales, los criterios para el análisis y mejoramiento de los 
indicadores de liquidez, actividad, y rentabilidad, y la utilización del análisis discriminante para diferenciar dos períodos establecidos, 2004 y 2009, permitieron concluir que los indicadores financieros: rentabilidad operativa del activo (RO), rotación de activo (RA), y nivel de endeudamiento (NE) del sector presentan diferencias significativas de un periodo a otro; y que, el apalancamiento a largo plazo (ALP) es el único indicador que mejora entre periodos.

Reyes y Briceño (2010) proponen un modelo financiero para el crecimiento corporativo sostenible, mediante cuantificaciones de componentes financieros como las ventas, la rentabilidad sobre el patrimonio (ROE), políticas de dividendos, de costos y de operaciones. El estudio determinó que la definición de políticas de gestión financiera aporta en la creación de valor de las empresas, en función del sector y mercado en el que opera. Se destaca la importancia de políticas de inversión y reinversión, dividendos, costos y de fijación de precios y comercialización, con la finalidad de generar valor y rentabilidad.

Briones et al., (2017) en su estudio sobre la gestión financiera desde la competitividad de las empresas bananeras en Ecuador, manejan un sistema de diagnóstico a través de indicadores financieros como liquidez, rentabilidad y apalancamiento, que son comparados con indicadores del sector. Se concluye, que evaluar constantemente la evolución de indicadores financieros es trascendental, pues permite evaluar los resultados de la gestión de forma específica y monitorear su evolución en el tiempo.

El análisis financiero, puede ser una herramienta, que, mediante el liderazgo y dirección de la gerencia, aporte a la acertada toma de decisiones para conseguir maximizar el valor empresarial; de forma que, las cualidades de la persona que administra una empresa repercuten en sus resultados. En este contexto, variables como el género y la formación académica, pueden influir en el desempeño empresarial.

En la actualidad, las organizaciones se enfrentan día a día a nuevos retos; por lo que, las características gerenciales pueden marcar la diferencia. La administración es la encargada de mejorar el desempeño laboral a través de promover actitudes y generar conocimiento, con el fin de obtener iniciativas innovadoras que potencien el talento de los miembros de la organización, la capacidad para tomar decisiones, la mejora continua y la gestión eficiente de recursos, para alcanzar un desarrollo eficiente y eficaz (Chávez et al., 2015)

Briozzo et al., (2017) en su estudio sobre Gobierno corporativo, financiamiento y género analizan la participación de mujeres en la alta gerencia de las empresas que participan en mercados de valores argentinos y si existe una relación entre el género y las decisiones de financiamiento. Con el uso de herramientas estadísticas, concluyen que cuando el gerente de una firma es mujer, existe mayor endeudamiento a corto plazo.

Para evaluar la hipótesis de que aquellas empresas con mayor inversión en la formación de sus directivos obtienen mejores resultados en cuanto a eficacia y rentabilidad, Aragón, et al., (2003) por medio de la aplicación de sucesivos análisis de regresión, con una base de datos recolectada a través de la realización de encuestas a 214 empresas españolas pequeñas y medianas, cuantifican el grado de aportación de la variable formación al éxito empresarial de las pequeñas y medianas empresas españolas. Los autores concluyen que en la muestra analizada 
hay indicios claros de una relación positiva entre la inversión en formación y los resultados empresariales en cuanto a rentabilidad y eficacia, a pesar de que su estudio de carácter exploratorio podría no ser concluyente para todas las compañías. En concordancia, Herrera et al., (2014) en su estudio sobre responsabilidad social, concluyen que la formación universitaria de los gerentes propietarios incide en el desempeño tanto financiero, como en prácticas de responsabilidad social de la compañía.

Por lo expuesto, es fundamental evaluar el desempeño empresarial, como aporte para identificar deficiencias y tomar medidas correctivas; así como, reconocer fortalezas y oportunidades en la gestión; además, del aporte que representan las cualidades de la gerencia en el desempeño económico de las firmas.

\section{Metodología}

La presente investigación corresponde a un estudio comparativo con un enfoque mixto de los cuatro principales sectores de la manufactura ecuatoriana: Cárnico (C1010), Textil (C1410), Bebidas (C11), y Muebles (C3100) codificados según la Clasificación Internacional Industrial Uniforme CIIU 4.

Se ha priorizado el estudio del sector manufacturero, considerando que, durante el año 2018, se caracterizó por ser el sector de mayor generación de Valor Agregado Bruto como aporte al Producto Interno Bruto del país con un 13,79\% (Banco Central del Ecuador, 2020). Dentro del sector manufacturero ecuatoriano, se han priorizado los subsectores que realizan los aportes más significativos en términos de personal empleado, activos totales, ventas, utilidad y número de empresas.

Tabla 1

Priorización de sectores a estudiar, año 2018

\begin{tabular}{llrrrrc}
\hline $\begin{array}{c}\text { Sector } \\
\text { económico }\end{array}$ & CIIU & Empleados & Activos & Ventas & Utilidad & $\begin{array}{c}\text { Número de } \\
\text { empresas }\end{array}$ \\
\hline Cárnico & C1010 & 16.496 & $1.089,53$ & 566,42 & $1.604,68$ & 99 \\
Bebidas & C11 & 11.160 & $1.281,98$ & 402,38 & $1.654,67$ & 197 \\
Textil & C1410 & 9.167 & 343,60 & 150,48 & 379,73 & 318 \\
Muebles & C3100 & 4.048 & 191,28 & 79,17 & 191,85 & 164 \\
\hline Total & & 40.871 & $2.906,38$ & $1.198,44$ & $3.830,93$ & 778 \\
$\begin{array}{l}\text { Porcentaje de } \\
\text { manufactura }\end{array}$ & $12,80 \%$ & $12,10 \%$ & $15,02 \%$ & $14,98 \%$ & $13,62 \%$ \\
\hline
\end{tabular}

Nota: Activos, ventas y utilidad expresados en millones de dólares.

La tabla 1 muestra la importancia de los subsectores de estudio dentro de manufactura, los cuales fueron priorizados con base en variables obtenidas de la información financiera que las sociedades reportaron en el año 2018 a la Superintendencia de Compañías, Valores y Seguros del Ecuador. 
El comportamiento de la gestión financiera fue estudiado mediante un análisis descriptivo de indicadores financieros (apéndice), y comparativo entre los sectores objeto de estudio. Se analizó la tendencia para describir su comportamiento en el periodo establecido entre los años 2013 al 2018. La información fue obtenida del estado de resultados y estado de situación financiera reportada por las firmas a la entidad de control. Además, se realizó un análisis de variables cualitativas como el género, la formación y afinidad académica, y la permanencia en el cargo de la gerencia.

Para llevar a cabo el procesamiento de los datos, se identificaron los valores atípicos que generan sesgo en los resultados según el criterio de Chauvenet, para posteriormente realizar pruebas de medias a través del test de Welch y prueba Anova para identificar diferencias significativas en las distintas variables de estudio.

\section{Resultados y Discusión}

A continuación, se muestran los resultados de la evaluación de la gestión financiera de los sectores Cárnico, Textil, Bebidas y Muebles en aspectos como liquidez, endeudamiento, actividad y rentabilidad, los cuales permiten visualizar el comportamiento de cada sector y compararlos entre sí mediante indicadores financieros que cuantifican su desempeño.

\section{Tabla 2}

Composición de la población analizada.

\begin{tabular}{ccc}
\hline Sector económico & Número de empresas & $\begin{array}{c}\text { Ingresos operacionales } \\
\text { (millones de dólares) }\end{array}$ \\
\hline Cárnico & 138 & $\$ 8.985,27$ \\
Bebidas & 166 & $\$ 6.187,78$ \\
Muebles & 139 & $\$ 1.365,14$ \\
Textil & 341 & $\$ 2.133,52$ \\
\hline Total & 784 & $\$ 18.671,71$ \\
\hline
\end{tabular}

La tabla 2 muestra la composición de la población analizada, en cuanto al número de empresas que han reportado actividad durante el período de estudio y los ingresos generados por estas firmas. El sector Textil se destaca con el mayor número de empresas; sin embargo, es el que menos ingresos ha generado durante el período de estudio. Los sectores Cárnico y Bebidas, registran un mayor volumen de ingresos operacionales, generados por un menor número de firmas.

El índice de liquidez mide la capacidad que tiene la empresa de hacer frente a sus obligaciones con terceros en el corto plazo; es decir, permite analizar la agilidad que tiene la empresa para cumplir con sus obligaciones corrientes. Este valor no debe ser excesivo, 
únicamente suficiente, con la finalidad de evitar sobreinversión en activos corrientes (Gitman y Zutter, 2012).

\section{Figura 1}

Liquidez corriente promedio de los sectores estudiados, 2013 - 2018

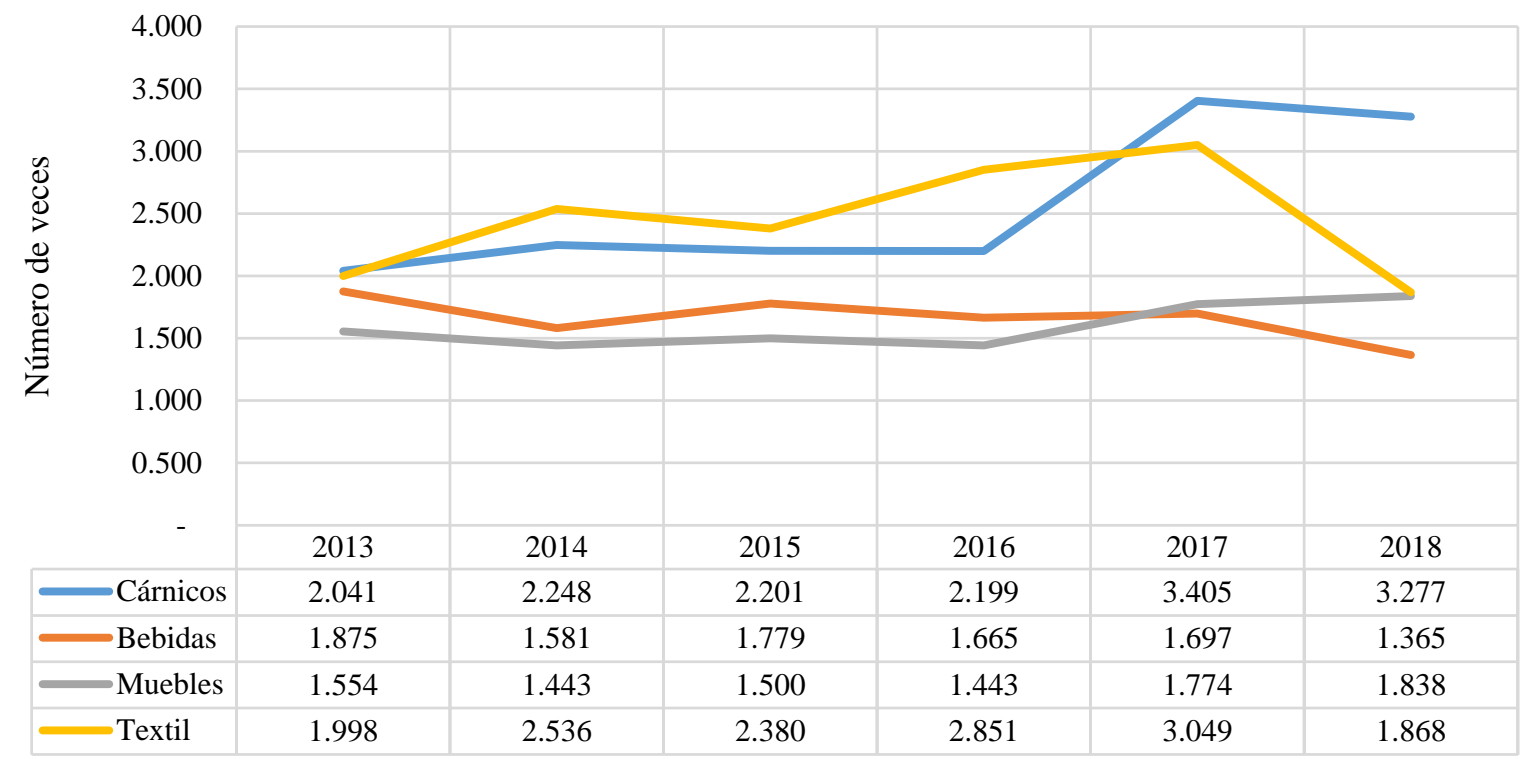

En la Figura 1 se observa la tendencia de los cuatro sectores analizados respecto a la liquidez presentada durante el período de estudio. El sector Textil muestra un mayor índice de liquidez en los primeros años con tendencia creciente; no obstante, para el año 2018 cae abruptamente hasta prácticamente igualar al sector Muebles. El sector Cárnico presentó una tendencia estable hasta el año 2016, a partir del cual se incrementa de manera considerable, que lo lleva a ser el sector con mayor liquidez dentro del periodo de estudio. Los sectores Bebidas y Muebles presentan una tendencia estable, con comportamientos contrarios en el último año.

La diferencia entre la liquidez corriente y la razón rápida recae en el inventario, rubro que está directamente ligado a la actividad que realiza cada subsector analizado. Por sus características, el sector Cárnico, al manejar productos perecibles, maneja un menor nivel de inventario; en contraste, Muebles y Textil presentan un mayor nivel de existencias. 


\section{Figura 2}

Liquidez corriente y razón rápida de los sectores estudiados, promedio 2013 - 2018

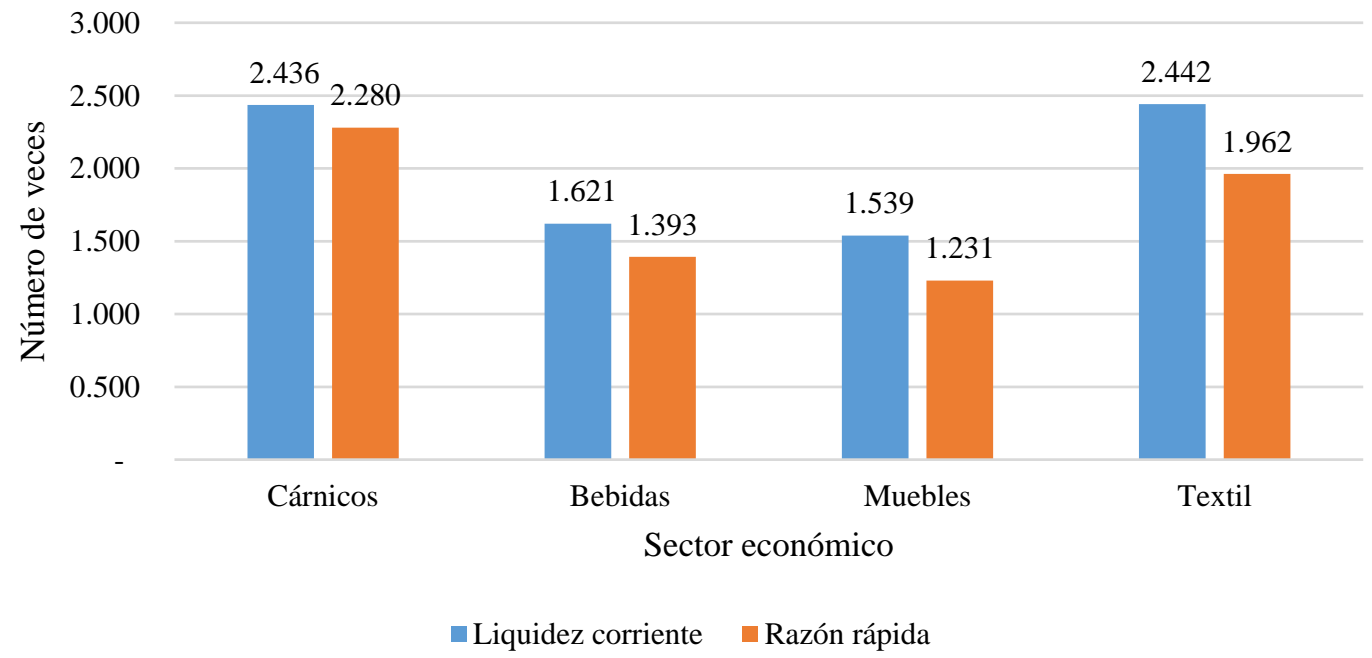

El análisis comparativo de la liquidez corriente y razón rápida de los cuatro sectores de estudio se evidencia en la Figura 2. Los sectores con mayor liquidez corriente promedio son Cárnico y Textil; sin embargo, la razón rápida es menor en el segundo sector mencionado, esto debido a que el sector Cárnico por su naturaleza maneja niveles de existencias menores; por ello, llega a ubicarse como el sector con mayor liquidez y con la menor dependencia en la realización de sus existencias.

El ciclo operativo considera los días requeridos para la venta de los inventarios y el periodo promedio de cobro, con el fin de obtener el tiempo promedio que un sector tarda en cumplir con el proceso completo de producción y venta de sus productos, incluido el cobro; es decir, hasta el que el inventario se convierte en efectivo.

\section{Figura 3}

Ciclo de operación promedio de los sectores estudiados, 2013 - 2018

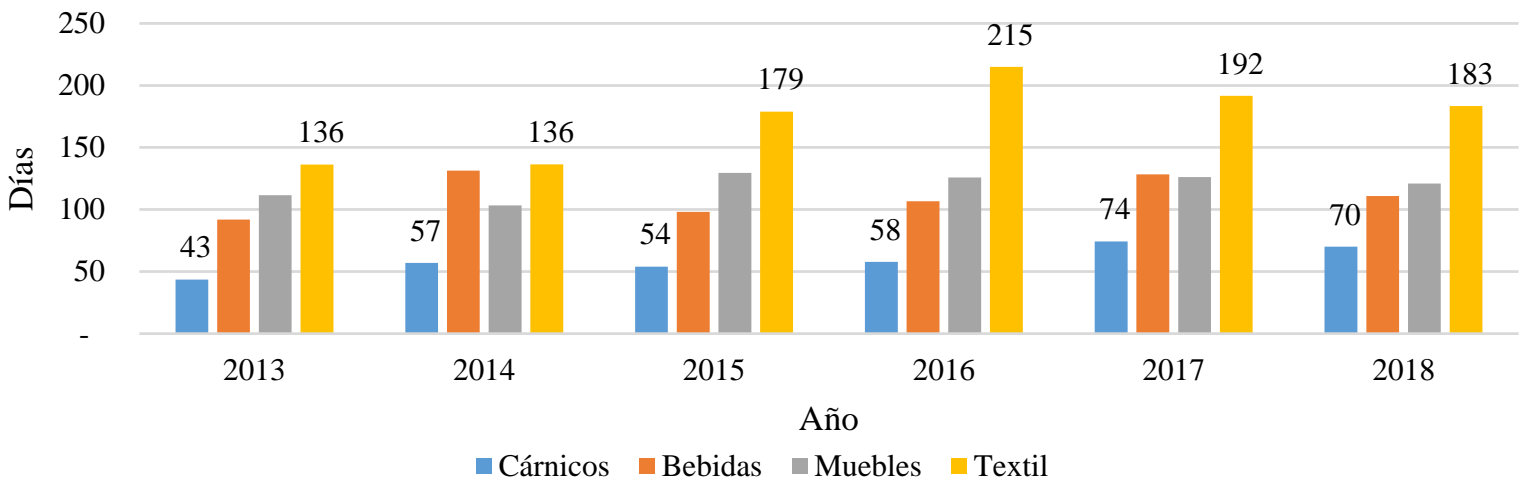


En la Figura 3 se observan comportamientos distantes entre los sectores de estudio, los cuales pueden estar explicados por las características propias de cada uno. Cárnico al ser un sector que maneja productos perecibles, los días que requieren para la venta de sus inventarios serán menores que los de otros sectores. En contraste, el sector Textil evidencia el ciclo operativo más alto. El sector Muebles, refleja un manejo eficiente de sus actividades, en contrapartida al sector Textil que a pesar de evidenciar mayor liquidez presenta un ciclo operativo mayor.

Cuando se hace referencia al ciclo de conversión de efectivo se considera el ciclo operativo disminuido por los días promedio de pago; es decir, refleja el tiempo promedio de un sector en convertir en efectivo la inversión realizada para la operación.

\section{Figura 4}

Ciclo de conversión de efectivo promedio de los sectores estudiados, 2013 - 2018.

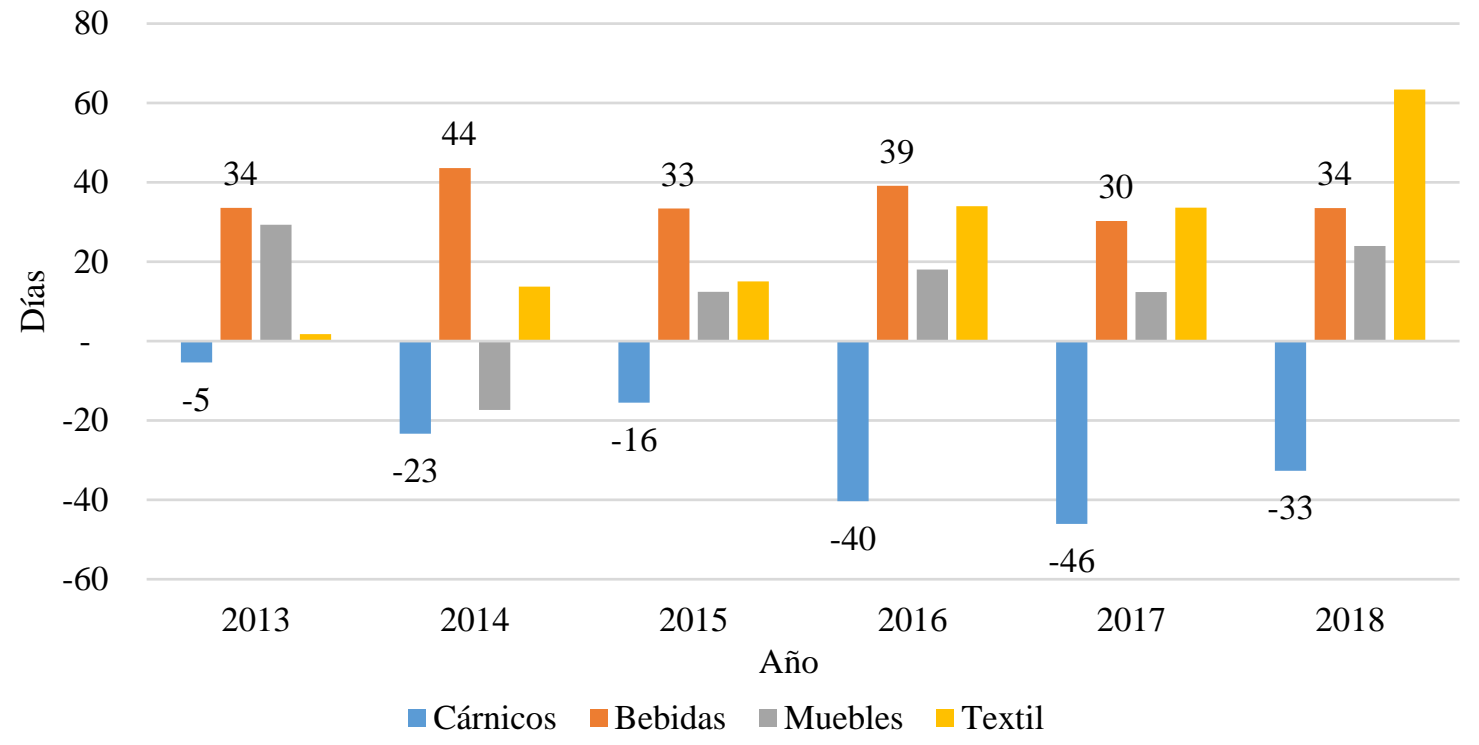

Dentro de la Figura 4 se muestra la ascendencia paulatina del sector Textil, lo que evidencia que, al analizar el ciclo operativo junto con el pago a proveedores da indicios de que el apalancamiento en estos en los primeros años de estudio fue bueno, situación que no se mantuvo en el tiempo. El sector Bebidas presenta un comportamiento similar en el periodo de estudio, ubicado como uno de los sectores que más tiempo demora en convertir su inversión en efectivo. El sector Cárnico, con un ciclo de efectivo negativo en todos los años, evidenció que los proveedores financian el ciclo del negocio de este sector, pues su apalancamiento en los proveedores es tan importante que podrían llegar a prescindir de otras fuentes de financiamiento de corto plazo. 
El índice de endeudamiento nos permite tener un acercamiento al financiamiento de los sectores a analizar; la finalidad es evaluar el grado de participación de los acreedores dentro de las empresas que forman el sector industrial.

\section{Figura 5}

Índice de endeudamiento de los sectores estudiados, promedio 2013 - 2018

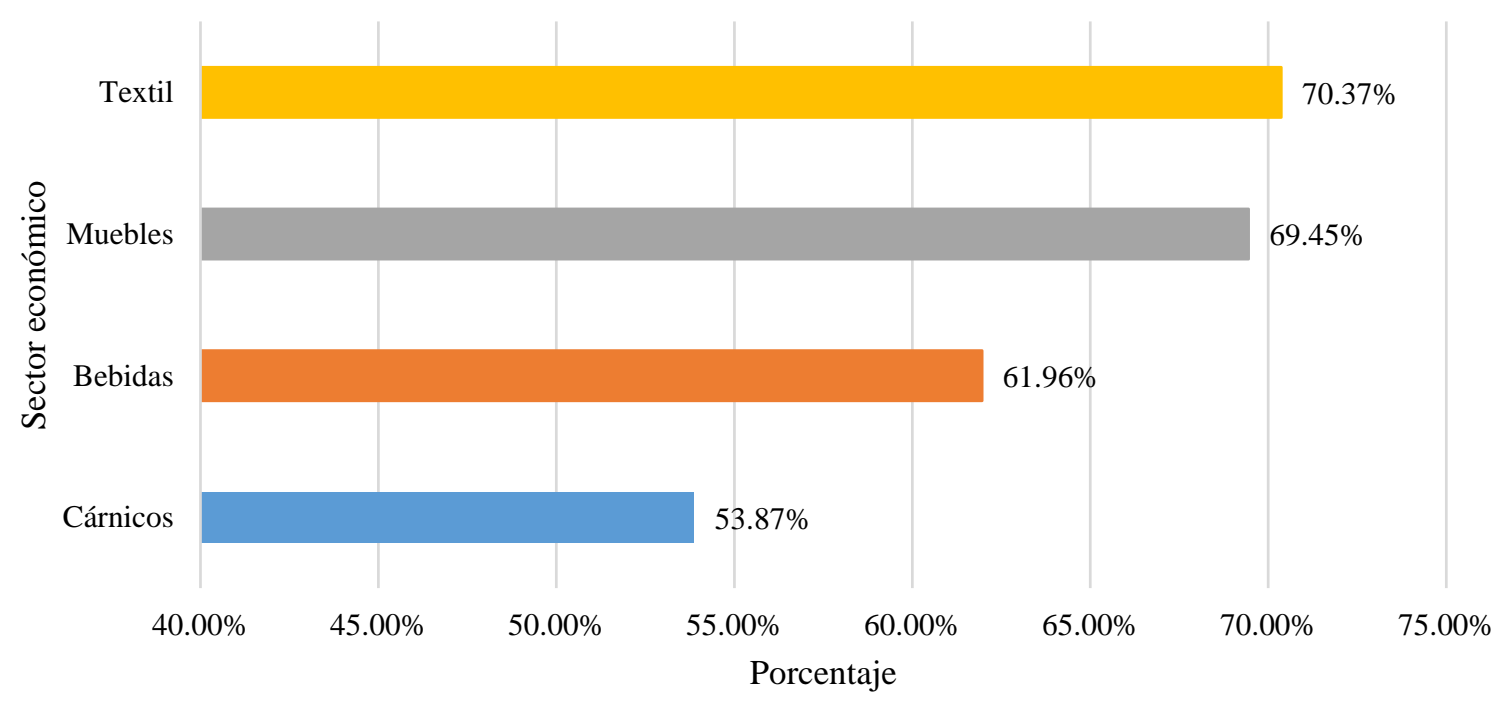

Se observa en la Figura 5 que todos los sectores analizados manejan un índice de endeudamiento mayor al 50\%, siendo el menor Cárnico, y evidenciando que los sectores que están relacionados con alimentos presentan un índice de endeudamiento menor. Resalta el sector Textil con el mayor índice de endeudamiento, debido a que durante la mayor parte del periodo de estudio se ubica como el sector más líquido, es también en promedio el más endeudado.

Al analizar la rentabilidad sobre activos se obtiene un indicativo de la sostenibilidad económica del sector industrial; este ratio indica la capacidad de generar ganancias mediante el aprovechamiento de los recursos. 


\section{Figura 6}

Rentabilidad sobre activos de los sectores estudiados, promedio 2013 - 2018.

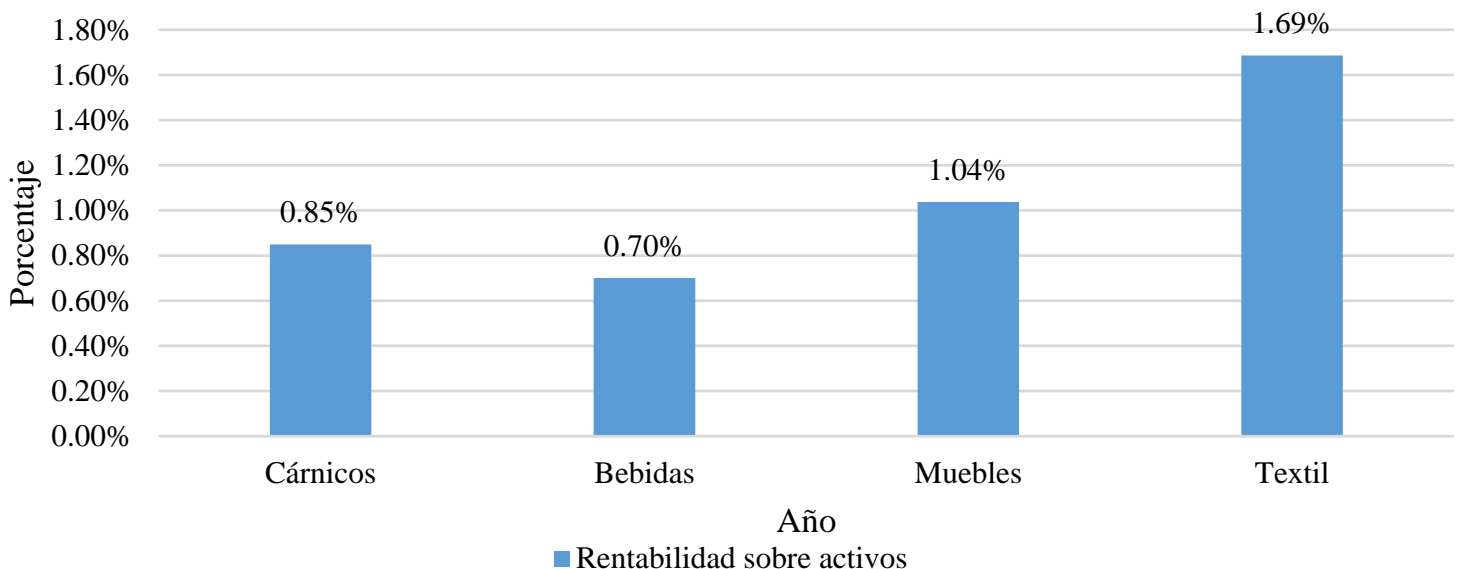

Al analizar la rentabilidad sobre activos, representada en la figura 6, se observa que de entre todos los analizados el sector Textil presenta una mayor rentabilidad sobre activos. Las industrias alimenticias estudiadas (Cárnico y Bebidas) son las que presentan índices menores de rentabilidad sobre los activos, siendo de menos del $1 \%$.

\section{Figura 7}

Rentabilidad sobre patrimonio de los sectores estudiados, promedio 2013 -2018.

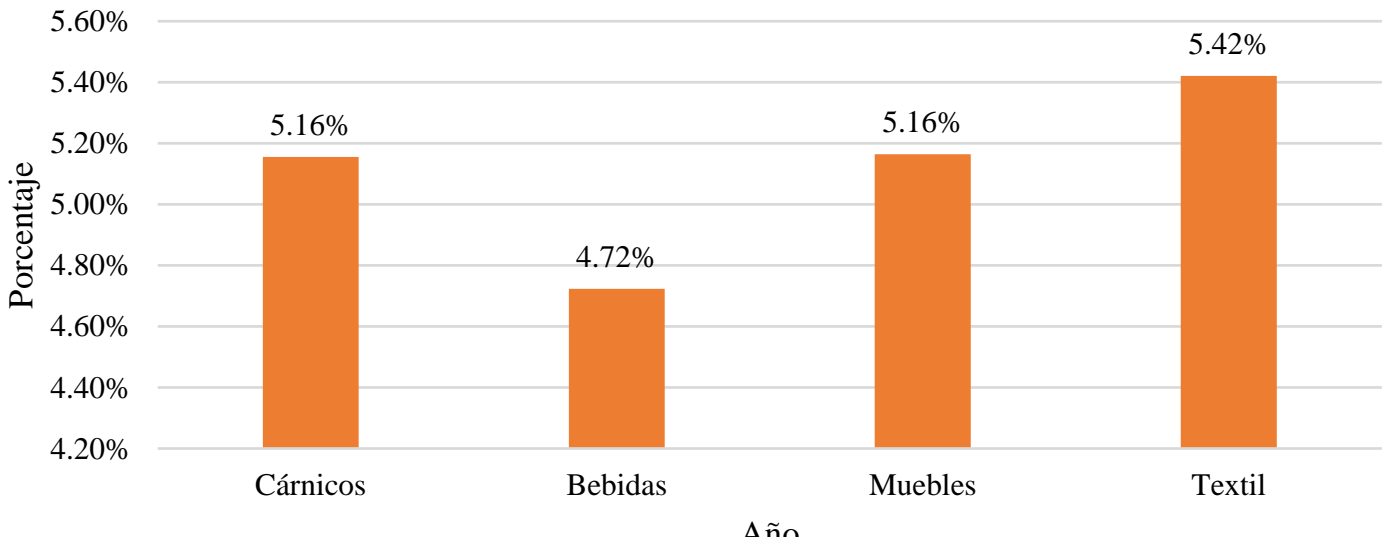

Rentabilidad sobre patrimonio

En cuanto a la rentabilidad sobre patrimonio representada en la figura 7, destaca el sector Textil en concordancia con el índice de rentabilidad anteriormente analizado. En su comportamiento conjunto el sector Textil presenta mayor liquidez, maneja un índice de 
endeudamiento alto, así como, su ciclo operativo requiere un mayor número de días promedio, no obstante, lidera los índices de rentabilidad sobre activos y sobre patrimonio. En cuanto al sector Bebidas, es el que menor rentabilidad presenta, tanto sobres activos como sobre el patrimonio.

A continuación, se analiza la evolución de la rentabilidad en los sectores estudiados que han presentado en promedio los mejores y peores resultados durante el período de estudio; a saber, el sector Bebidas y Textil.

\section{Figura 8}

Rentabilidad sobre activos de los sectores: Bebidas y Textil, medianas 2013 - 2018

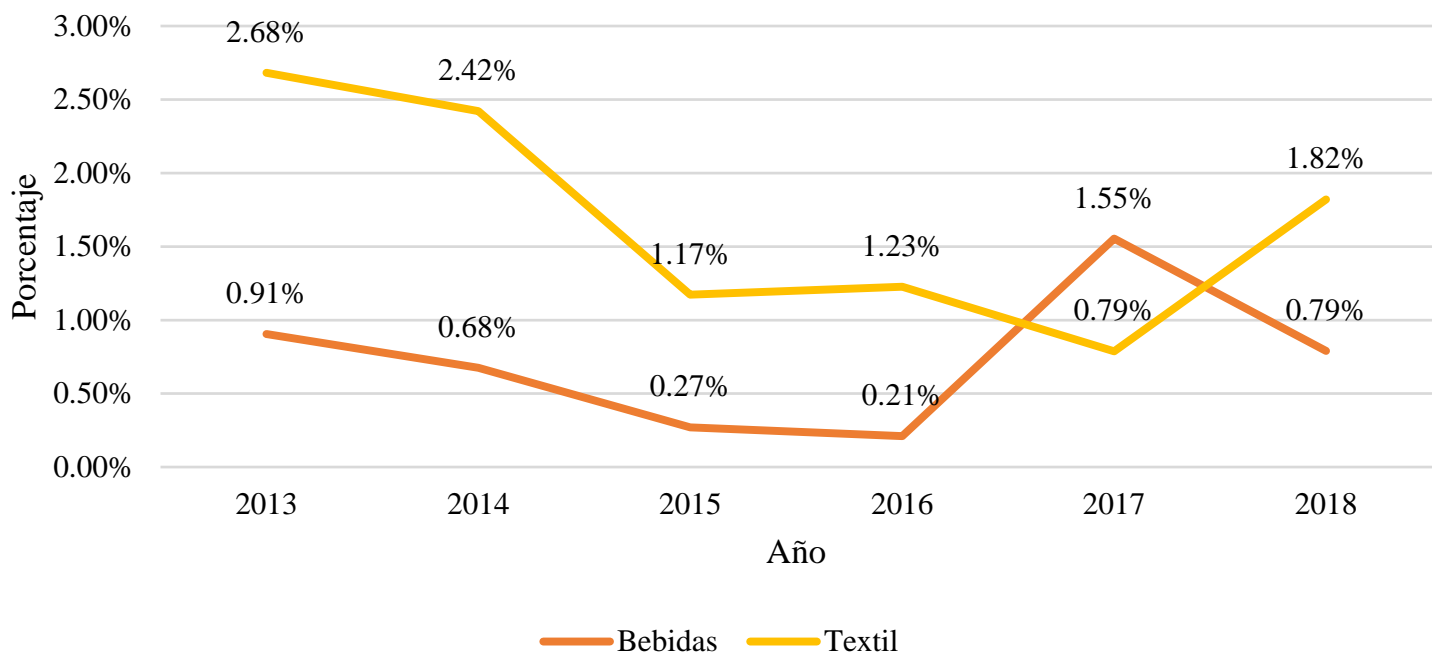

$\mathrm{Al}$ analizar el rendimiento sobre los activos, expuesto en la figura 8 , se observa que el sector Textil, a pesar de mostrar mayor rentabilidad en los primeros años de estudio, tiene una tendencia a la baja que se revierte para el último año, a diferencia del sector Bebidas, que presenta una tendencia decreciente con una efímera recuperación en el año 2017.

En cuanto a la rentabilidad sobre el patrimonio, se utiliza para evaluar la capacidad que tienen las empresas que forman del sector para generar beneficios a partir de la inversión que realizan los socios. 


\section{Figura 9}

Rentabilidad sobre patrimonio de los sectores: Bebidas y Textil, medianas 2013 - 2018

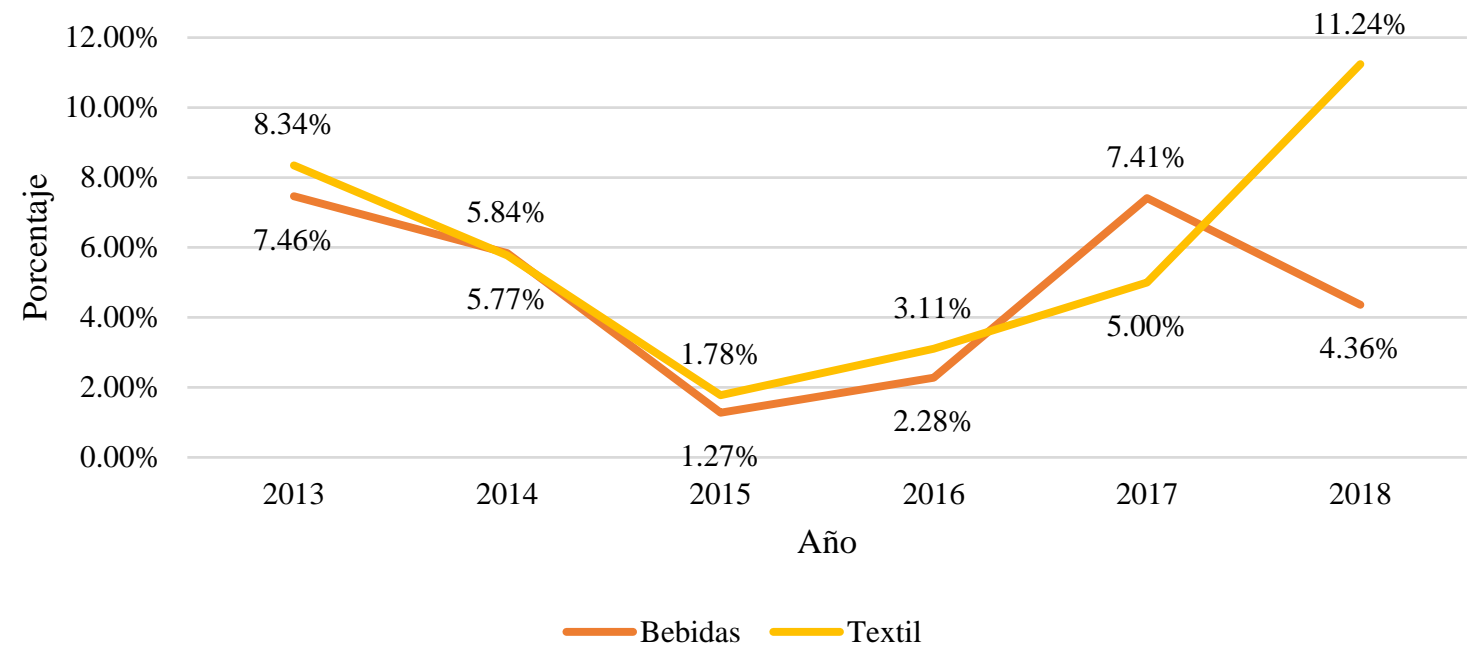

En la figura 9 se observa que los sectores Bebidas y Textil han presentado tendencias similares durante los primeros cinco años analizados, con un decrecimiento inicial seguido de una recuperación; sin embargo, se destaca que, en el caso del sector Textil, esta recuperación se mantuvo de forma sostenida hasta 2018; mientras que, en el sector Bebidas, no se mantuvo esta tendencia, la cual se revierte para 2018.

Las cualidades de los gerentes de las empresas que conforman los sectores económicos objeto de estudio son presentadas con la finalidad de describir la situación actual, y establecer nexos entre las cualidades de la gerencia y el desempeño de las firmas.

\section{Tabla 3}

Género de la gerencia

\begin{tabular}{lcccc}
\hline Sector & \multicolumn{2}{c}{ Masculino } & \multicolumn{2}{c}{ Femenino } \\
\cline { 2 - 5 } económico & Frecuencia & Porcentaje & Frecuencia & Porcentaje \\
\hline Cárnico & 79 & $76,7 \%$ & 24 & $23,3 \%$ \\
Bebidas & 81 & $80,2 \%$ & 20 & $19,8 \%$ \\
Muebles & 61 & $76,2 \%$ & 19 & $23,8 \%$ \\
Textil & 134 & $62,0 \%$ & 82 & $38,0 \%$ \\
\hline
\end{tabular}

Como se puede observar en la tabla 3, se evidencia una participación intensiva del género masculino en la gerencia. El sector industrial que presenta mayor porcentaje de mujeres en la gerencia es el Textil; sin embargo, su incidencia es menor al 50\%. 


\section{Tabla 4}

Formación académica de la gerencia

\begin{tabular}{lcccc}
\hline \multirow{2}{*}{$\begin{array}{c}\text { Sector } \\
\text { económico }\end{array}$} & \multicolumn{2}{c}{ Bachillerato } & \multicolumn{2}{c}{ Universitaria } \\
\cline { 2 - 5 } Cárnico & 50 & $54,4 \%$ & 42 & Precuencia \\
Bebidas & 34 & $37,8 \%$ & 56 & $45,6 \%$ \\
Muebles & 35 & $48,0 \%$ & 38 & $62,2 \%$ \\
Textil & 116 & $59,8 \%$ & 78 & $52,0 \%$ \\
\hline
\end{tabular}

Una cualidad diferencial en el desempeño de la administración y por ende en los resultados de la empresa es la formación académica de la gerencia. Se puede observar en la tabla 4, que este comportamiento depende del sector al que pertenecen las empresas, siendo el sector Textil el que presenta un mayor porcentaje de gerentes con formación de bachillerato; en contrapartida, la gerencia de las empresas del sector Bebidas, poseen en mayor porcentaje formación universitaria. Si bien, 78 gerentes del sector Textil poseen formación universitaria, 25 de ellos no tienen una formación afín, como se observa en la tabla 5.

\section{Tabla 5}

Afinidad de la formación académica de la gerencia

\begin{tabular}{ccccc}
\hline \multirow{2}{*}{$\begin{array}{c}\text { Sector } \\
\text { económico }\end{array}$} & Frecuencia & Porcentaje & Frecuencia & Porcentaje \\
\cline { 2 - 5 } Cárnico & 34 & $81,0 \%$ & 8 & $19,0 \%$ \\
Bebidas & 40 & $71,4 \%$ & 16 & $28,6 \%$ \\
Muebles & 29 & $76,3 \%$ & 9 & $23,7 \%$ \\
Textil & 53 & $68,0 \%$ & 25 & $32,0 \%$ \\
\hline
\end{tabular}

La mayor parte de profesionales universitarios que se encuentran en la gerencia de las empresas objeto de estudio, cuentan con una formación afín al sector económico en el que se desempeñan o a las ciencias económicas y administrativas; se destaca el sector Cárnico como aquel con un mayor porcentaje de formación afín.

\section{Tabla 6}

Permanencia en el cargo de la gerencia en años

\begin{tabular}{|c|c|c|c|c|c|}
\hline \multicolumn{2}{|c|}{ Categoría } & \multicolumn{4}{|c|}{ Porcentaje } \\
\hline Desde & Hasta & Cárnico & Bebidas & Muebles & Textil \\
\hline 0 & 6,5 & $59,7 \%$ & $66,3 \%$ & $61,2 \%$ & $84,2 \%$ \\
\hline 6,5 & 13 & $27,5 \%$ & $18,8 \%$ & $28,8 \%$ & $7,9 \%$ \\
\hline 13 & 19,5 & $7,8 \%$ & $10,9 \%$ & $6,3 \%$ & $5,1 \%$ \\
\hline 19,5 & 26 & $2,0 \%$ & $4,0 \%$ & $2,5 \%$ & $2,3 \%$ \\
\hline 26 & 32,5 & $3,0 \%$ & $0,00 \%$ & $1,2 \%$ & $0,5 \%$ \\
\hline
\end{tabular}


En la Tabla 6 se visualiza la permanencia en el cargo de la gerencia medida en años. En todos los sectores más del 50\% de gerentes se encuentran, en promedio, en su cargo hasta 6,5 años; además, menos del 5\% ocupan su cargo por más de 19,5 años, lo que demuestra que con el tiempo las empresas y sus inversionistas buscan la renovación de la administración, con gerentes con ideas y objetivos nuevos. El sector que mantiene por períodos más prolongados a sus administradores es Bebidas, cuyo porcentaje de gerentes que mantienen el cargo por más de 13 años es de $14,9 \%$.

Se analizaron los resultados de la gestión financiera de las empresas de estudio relacionándolas con las cualidades de la gerencia, con el objetivo de determinar si estas inciden en el desempeño empresarial.

\section{Tabla 7}

Género de la gerencia e índice de endeudamiento

\begin{tabular}{lcc}
\hline Sector económico & Masculino & Femenino \\
\hline Cárnico ** & $51,67 \%$ & $62,48 \%$ \\
Bebidas & $65,13 \%$ & $64,08 \%$ \\
Muebles & $68,90 \%$ & $67,87 \%$ \\
Textil ** & $64,48 \%$ & $71,92 \%$ \\
\hline
\end{tabular}

**Diferencia estadísticamente significativa al 0,05 para test de Welch

En la Tabla 7 se analiza el índice de endeudamiento de los distintos sectores industriales junto con el género de la gerencia. Se evidencia que las empresas de los sectores Cárnico y Textil que son gerenciadas por personas con género femenino, tienen un mayor nivel de endeudamiento, siendo más propensas al riesgo y a utilizar el apalancamiento en recursos de terceros como estrategia de generación de valor en mayor medida. Estos resultados están alineados con el estudio de Briozzo et al., (2017) quienes demuestran que las empresas gerenciadas por mujeres optan por una estrategia de gestión financiera agresiva.

\section{Tabla 8}

Formación académica de la gerencia e índice de endeudamiento

\begin{tabular}{lccc}
\hline \multicolumn{2}{l}{ Sector económico } & Bachillerato & Universitaria \\
\hline Cárnico & $* * *$ & $50,44 \%$ & $60,22 \%$ \\
Bebidas & $* *$ & $62,04 \%$ & $65,52 \%$ \\
Muebles & $* *$ & $68,83 \%$ & $69,28 \%$ \\
Textil & $6 *$ & $67,20 \%$ & $69,25 \%$ \\
\hline
\end{tabular}

** Diferencia estadísticamente significativa al 0,05 para test de Welch

*** Diferencia estadísticamente significativa al 0,01 para test de Welch

La formación académica y su incidencia en el endeudamiento se presenta en la tabla 9. Se evidenció que las empresas gerenciadas por profesionales tienen un mayor índice de 
endeudamiento frente a aquellas administradas por bachilleres; esto quiere decir, que los gerentes con estudios universitarios presentan mayor propensión al riesgo y una tendencia a apalancar las operaciones empresariales, en mayor medida, con recursos de terceros.

\section{Tabla 9}

Formación académica de la gerencia y liquidez corriente

\begin{tabular}{|c|c|c|c|}
\hline \multicolumn{2}{|c|}{ Sector económico } & Bachillerato & Universitaria \\
\hline Cárnico & & 2,424 & 3,034 \\
\hline Bebidas & $* * *$ & 1,948 & 1,341 \\
\hline Muebles & $* *$ & 1,725 & 1,531 \\
\hline Textil & $* *$ & 2,183 & 2,902 \\
\hline
\end{tabular}

Herrera et al., (2014) afirma la incidencia que puede tener la formación académica de la gerencia en el desempeño empresarial.

En el caso de la formación académica y su incidencia en la liquidez corriente, que se presenta en la Tabla 9, se evidencia que los mayores niveles se encuentran, en promedio, en las empresas de los sectores Textil y Cárnico, cuando estas son gerenciadas por profesionales universitarios.

\section{Tabla10}

Afinidad de la formación académica de la gerencia y liquidez corriente

\begin{tabular}{|c|c|c|}
\hline Sector económico & Afín & No afín \\
\hline Cárnico & 3,386 & 2,329 \\
\hline Bebidas $* *$ & 1,467 & 1,047 \\
\hline Muebles $* *$ & 1,649 & 1,616 \\
\hline Textil & 2,921 & 2,268 \\
\hline
\end{tabular}

** Diferencia estadísticamente significativa al 0,05 para test de Welch

Cuando la liquidez corriente se estudia junto con la afinidad de la formación, según la Tabla 10, se pudo observar mayores índices en las empresas cuyos gerentes tienen una formación afín independiente de la actividad de la empresa; por lo que, esta situación es la misma en los cuatro sectores analizados, demostrando que los profesionales con formación afín al sector o a los negocios manejan de manera más efectiva las obligaciones en el corto plazo.

Los resultados del presente estudio ratifican la importancia de la gestión financiera en el desempeño empresarial, en concordancia con lo expuesto por Portal, et al., (2016); además, de la importancia de que los administradores dispongan de indicadores financieros que les permitan 
evaluar los resultados empresariales respecto a los de la industria, criterio afirmado por Bernal y Amat, (2012), Reyes y Briceño, (2010) y Briones et al., (2017).

\section{Conclusiones y Recomendaciones}

La presente investigación evaluó la gestión financiera de los cuatro sectores económicos más importantes de la industria manufacturera del Ecuador, lo cual ha permitido conocer las características del desempeño económico de estos sectores.

Se evidenció durante el período de estudio que el sector Textil y Cárnico mantuvieron mayores niveles de liquidez; a la vez que, todos los sectores analizados están en capacidad de cubrir sus obligaciones corrientes a pesar de no realizar sus existencias. Todos los sectores analizados han evidenciado un deterioro del ciclo de operación; a pesar de aquello, el sector Cárnico presenta la mayor eficiencia operativa y las mejores condiciones otorgadas por parte de proveedores. En cuanto al endeudamiento, los resultados indican que los cuatro sectores apuestan por una estructura de financiamiento en la que destaca prioritariamente el uso de recursos de terceros por sobre los recursos propios. Esta situación derivó en que todos los sectores presenten índices de rentabilidad positivos, aunque marcados por volatilidad en función del entorno macroeconómico inestable en el que se desenvuelven.

El análisis cualitativo evidenció la participación mayoritaria del género masculino en la gerencia empresarial de los sectores estudiados. La mayoría de los gerentes de las empresas de los sectores estudiados, cuentan con formación académica de bachillerato, escenario que puede deberse a la influencia del emprendimiento por oportunidad en el tejido empresarial ecuatoriano.

En cuanto a la incidencia de las cualidades de la gerencia en el desempeño económico de las firmas, se encontró que las empresas gerenciadas por mujeres presentaron mayores niveles de endeudamiento, lo cual se puede relacionar con un estilo de administración que contempla estrategias de financiamiento agresivas con la finalidad de maximizar el valor empresarial a la par con una mayor propensión al riesgo. Un escenario similar se encuentra en las empresas gerenciadas por personas con formación académica universitaria, las cuales financian sus activos con recursos de terceros en mayor proporción, lo que se puede explicar debido a la mayor capacidad técnica de identificar con precisión el riesgo inherente a la actividad empresarial. Situación concordante con las empresas cuyos gerentes poseen formación afín al sector industrial o a las ciencias económicas y administrativas, que presentaron mayores niveles de liquidez.

\section{Referencias bibliográficas}

Aragón Sánchez, A., Sanz Valle, R., y Barba Aragón, M. I. (2003). Efectos de la formación de directivos en las Pymes españolas. Estudios Financieros. Revista de Trabajo y Seguridad Social: Comentarios, Casos Prácticos : Recursos Humanos, 243, 103-135.

Banco Central del Ecuador. (2020). Boletín de cuentas nacionales trimestrales. Presentación Informativa de Resultados a Precios Constantes de 2007, 2020.IT. https://contenido.bce.fin.ec/home1/estadisticas/cntrimestral/CNTrimestral.jsp 
Bernal, D., y Amat, O. (2012). Anuario de ratios financieros sectoriales en México para análisis comparativo empresarial. Ra Ximhai, 8(2), 267-281.

Besley, S., y Brigham, E. (2001). Fundamentos de Administración Financiera (Segunda Ed). McGraw Hill.

Briones, R., Morales, F., y Bajaña, F. (2017). Financial management from the competitiveness of the agriculture of a banana group in the provinces of Guayas and Los $R^{\prime}$ los. 2(8), 1621.

Briozzo, A., Albanese, D., y Santolíquido, D. (2017). Gobierno corporativo, financiamiento y género: un estudio de las pymes emisoras de títulos en los mercados de valores argentinos. Contaduría y Administración, 62(2), 339-357. https://doi.org/10.1016/j.cya.2017.01.005

Chávez, S., De las Salas, M., y Bozo Acosta, R. (2015). Competencias gerenciales y desempeño laboral en empresas aseguradoras. CICAG: Revista Del Centro de Investigación de Ciencias Administrativas y Gerenciales, 12(2), 178-196.

Córdoba Padilla, M. (2012). Gestión Financiera (Segunda Ed). ECOE Ediciones.

Danvers, K., y Oliver, T. (2013). Using financial models as a value-added management tool. Strategic Finance, 95(9), 47-51.

Gitman, L., y Zutter, C. (2012). Principios de administración financiera. In Pearson Education (Vol. 12). Pearson Education.

Global Entrepreneurship Monitor; ESPAE. (2020). Global Entrepreneurship Monitor Ecuador 2019-2020 (Vol. 8, Issue 2).

Herrera, J., Larrán Jorge, M., Lechuga Sancho, M. P., y Martínez- Martínez, D. (2014). Motivaciones hacia la Responsabilidad Social en las PYMEs familiares. European Journal Of Family Business, 4(1), 21-44. https://doi.org/10.24310/ejfbejfb.v4i1.5037

Instituto Nacional de Estadísticas y Censos. (2020). Encuesta Nacional De Desempleo Y Subempleo. In Encuesta Nacional de empleo, desempleo y subempleo. Indicadores Laborales Diciembre 2019. https://www.ecuadorencifras.gob.ec/documentos/webinec/EMPLEO/2019/Junio/201906_Mercado_Laboral_final.pdf

Masilo, A., y Gómez Aguirre, M. (2016). Estudio de revisión sobre la planeación financiera y propuesta de modelo empírico para pymes de México. Cimexus, 11(2), 73-106.

Morelos, J., Fontalvo, T., y de la Hoz, E. (2012). Análisis de los Indicadores Financieros en las Sociedades Portuarias de Colombia. Entramado, 8(1), 14-26.

Nava, M. A. (2009). Análisis financiero: una herramienta clave para una gestión financiera eficiente. Revista Venezolana de Gerencia, 14(48), 606-628.

Pacheco, J., Castañeda, W., y Caicedo, C. (2002). Indicadores Integrales de Gestión. Mc Graw Hill.

Paramasivan, C; Subramian, T. (2012). Financial Management (Quinta Edi). New Age International.

Pardo, S., y Peña, Á. R. (2018). La contabilidad financiera como motor de la redefinición de la concepción de empresa. Cuadernos de Contabilidad, 18(46). https://doi.org/10.11144/javeriana.cc18-46.cfmr

Portal, M., Feitó, D., Bernal E., B. (2016). Evaluación de la gestión económico-financiera en microempresas mexicanas. FAEDPYME International Review - FIR, 5, 20-31.

Ramírez-Urquidy, M., Aguilar-Barceló, J. G., y Portal-Boza, M. (2018). O impacto das práticas de gestão econômico-financeira no desempenho de microempresas Mexicanas: Uma análise multivariada. Revista Brasileira de Gestao de Negocios, 20(3), 319-337. https://doi.org/10.7819/rbgn.v20i3.3518

Esta obra se comparte bajo la licencia Creative Common Atribución-No Comercial 4.0 International (CC BY-NC 4.0) 
Reyes, G. E., y Briceño, A. (2010). Propuesta de un modelo financiero para el crecimiento corporativo sostenible. Finanzas y Política Económica, 2.

Superintendencia de Compañías Valores y Seguros del Ecuador. (2020). Superintendencia de Compañias, Valores y Seguros del Ecuador. https://www.supercias.gob.ec/portalscvs/

Terrazas, R. (2016). Modelo De Gestión Financiera Para Una Organización. Perspectivas, 23(3), 55-72.

Van Horne, J., y Wachowicz, J. (2010). Fundamentos de Administración Financiera (Décimoterc). Pearson Education.

Zabukovec, A., y Jaklič, J. (2015). The Impact of Information Visualisation on the Quality of Information in Business Decision-Making. International Journal of Technology and Human Interaction, 11(2), 61-79. https://doi.org/10.4018/ijthi.2015040104

Zambrano, J., y Lasio, V. (2019). Jóvenes emprendedores en Ecuador 2012 - 2017. In Global Entrepreneurship Monitor.

\section{Apéndice 1}

Razones financieras aplicadas

\begin{tabular}{|c|c|c|}
\hline Descripción & Cálculo & Autor \\
\hline Liquidez corriente & $\begin{array}{l}\text { Activos corrientes / Pasivos } \\
\text { corrientes }\end{array}$ & Gitman y Zutter \\
\hline Razón rápida & $\begin{array}{l}\text { (Activos corrientes - inventario } \\
\text { productos terminados) / Pasivos } \\
\text { corrientes }\end{array}$ & Gitman y Zutter \\
\hline Ciclo Operativo & $\begin{array}{l}\text { Periodo Promedio de Inventario + } \\
\text { Periodo Promedio de Cobro }\end{array}$ & Van Horne y Wachowicz \\
\hline $\begin{array}{l}\text { Ciclo de Conversión de } \\
\text { Efectivo }\end{array}$ & $\begin{array}{l}\text { Periodo Promedio de Inventario } \\
\text { +Periodo Promedio de Cobro - } \\
\text { Periodo Promedio de Pago }\end{array}$ & Van Horne y Wachowicz \\
\hline $\begin{array}{l}\text { Índice de } \\
\text { endeudamiento }\end{array}$ & Total de pasivos / Total de activos & Gitman y Zutter \\
\hline $\begin{array}{c}\text { Rendimiento sobre } \\
\text { activos totales (ROA) }\end{array}$ & Utilidad neta / Total de activos & Gitman y Zutter \\
\hline $\begin{array}{l}\text { Rendimiento sobre el } \\
\text { patrimonio (ROE) }\end{array}$ & Utilidad neta / Total de patrimonio & Gitman y Zutter \\
\hline
\end{tabular}

